\section{Fortalecimiento de la (auto)gestión \\ de cooperativas de trabajo en el marco \\ de la Economía Social y Solidaria en la ciudad de Santa Fe}

\author{
Julio Tealdo \\ Docente investigador de la Facultad \\ de Ciencias Jurídicas y Sociales \\ Universidad Nacional del Litoral \\ (UNL), Argentina. \\ Director del Programa de Economía \\ Social y Solidaria, Secretaría de \\ Extensión UNL

\section{Orlando Sotto} \\ Docente de la Facultad de Ciencias \\ Jurídicas y Sociales, UNL. \\ Integrante del Programa de Economía \\ Social y Solidaria, Secretaría de \\ Extensión UNL.
}

\author{
Eugenio Serafino \\ Docente de la Facultad de Ciencias \\ Económicas, UNL. \\ Integrante del Programa de Economía \\ Social y Solidaria, Secretaría de \\ Extensión, UNL
}

Economía Social y Solidaria /

Intervenciones

RECEPCIÓN: 26/06/15

ACEPTACIÓN FINAL: 27/07/15

\section{Resumen}

La forma de autogestión de las cooperativas de trabajo como modo de organización y administración de la producción y distribución genera una serie de inconvenientes que obstaculizan su fortalecimiento. Conciliar esto con el refuerzo de la democracia participativa fue un gran desafío para las cooperativas de trabajo y el equipo extensionista del Proyecto de Extensión de Interés Social (PEIS) "Fortalecimiento y capacitación de las cooperativas de trabajo y otras organizaciones sociales en el marco de la Economía Social y Solidaria en la ciudad de Santa Fe" de la Universidad Nacional del Litoral (UNL).

En los encuentros realizados, los asociados de las cooperativas no sólo obtuvieron capacidades y herramientas de gestión nuevas, sino que también despertaron en su interior la voluntad necesaria para seguir adquiriendo otras habilidades y para replicar estos conocimientos en su comunidad.

\section{Palabras clave}

- cooperativa de trabajo

- Economía Social y Solidaria

- autogestión

- administración

\section{Resumo}

A forma de autogestão das cooperativas de trabalho como forma de organização e administração da produção e distribução, gera uma série de inconvenientes que dificultam seu fortalecimento. Conciliar isso com o fortalecimento da democracia participativa foi um grande desafio para as cooperativas de trabalho e o equipe de extensão do Projeto de Extensão de Interesse Social (PEIS)."Fortalecimento e capacitação das cooperativas de trabalho e outras organizações sociais no contexto da Economia Social e Solidária na cidade de Santa Fe".

Nos encontros realizados, os membros das cooperativas não só alcanzaram capacidades e ferramentas de gestão novas, senão que também almejaram uma vontade interior necessária para continuar adquirindo outras habilidades e levar esses conhecimentos à sua comunidade

Palavras chave

- cooperativa de trabalho

- Economia Social e Solidaria

- autogestão

- administração
Para citación de este artículo

Tealdo, J.; Sotto, O. y Serafino, E. (2015). Fortalecimiento de la (auto)gestión de cooperativas de trabajo en el marco de la Economía Social y Solidaria en la ciudad de Santa Fe. En Revista +E versión digital, (5), pp. 188-195. Santa Fe, Argentina: Ediciones UNL. 


\section{6}

la propuesta de articular acciones

con cooperativas de trabajo surge

de la relevante función social

que tienen como creadoras de

trabajo genuino y digno

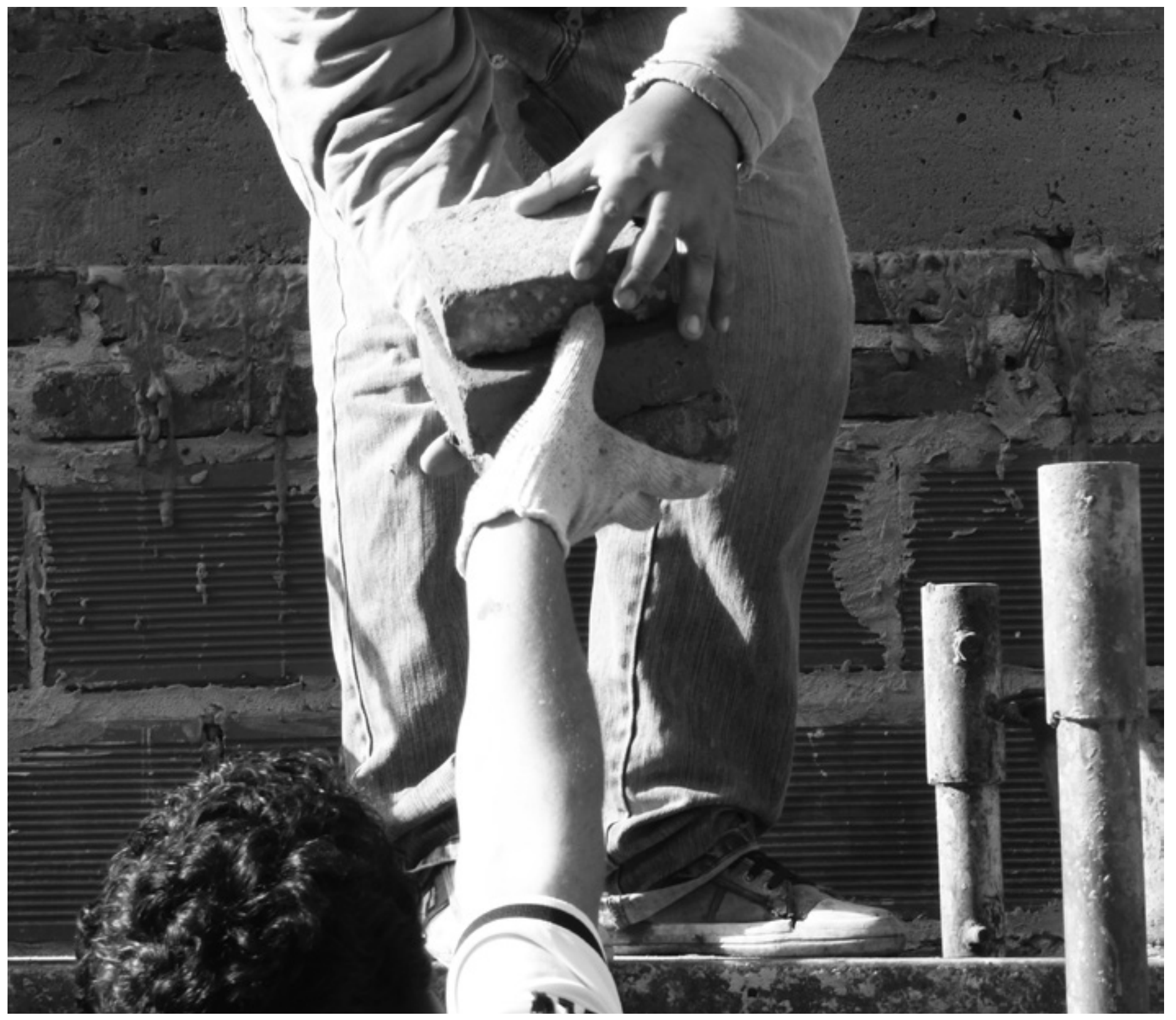




\section{Introducción}

Desde los albores de lo que entendemos por Revolución Industrial, la organización cooperativa se conformó como un conjunto de prácticas que permiten resolver de forma asociativa situaciones de necesidad que padecen hombres y mujeres en estado de vulnerabilidad frente a la ofensiva del sistema económico capitalista. Es así que en el siglo XIX surgió el movimiento cooperativo como una alternativa emancipadora orientada a construir un modo de socialización basado en una cultura distinta, en la que los valores de solidaridad, ayuda mutua y participación, sirvan como guía para la conformación de una sociedad más justa e igualitaria.

Los emprendimientos colectivos pueden adquirir distintas formas; sin embargo, nuestro trabajo se ha focalizado en el cooperativismo de trabajo dada la importancia que ha tomado esta forma asociativa durante los últimos años.

Vale recordar que en las últimas décadas del siglo XX el despliegue de políticas económicas neoliberales provocó en Argentina y, particularmente, en la ciudad de Santa Fe, un fuerte impacto en términos de exclusión, marginación social y desempleo. En este contexto, el cooperativismo de trabajo fue practicado en forma creciente con la iniciativa de trabajadores desocupados o precarizados, conformándose como una alternativa real a la situación de empleo asalariado deficitario.

La propuesta de articular acciones con cooperativas de trabajo surge de la relevante función social que tienen como creadoras de trabajo genuino y digno, como centros productivos y sociales aglutinadores de personas que muchas veces no encuentran su lugar en el mercado y como núcleo de referencia e influencia en los lugares donde residen y trabajan. Son entidades generadoras de sociedad y no de utilidad.

Si nos enfocamos en las características propias de una cooperativa de trabajo, podemos definirla como una asociación de personas que se reúnen para trabajar de manera conjunta, en la que cada asociado pone a disposición de la organización su trabajo (en lugar de su capital), con la finalidad de que los resultados del trabajo colectivo garantice el medio de vida de cada uno de los trabajadores y el de sus familias, mejorando así su situación social y económica, dejando de ser trabajadores asalariados para así transformarse en dueños de su emprendimiento colectivo. Siguiendo a Cracogna (2002), el aspecto que distingue las cooperativas de trabajo del resto radica en su objeto social específico que consiste en brindar ocupación (trabajo) a sus asociados.

Según el Instituto Nacional de Asociativismo y Economía Social (INAES), hasta el año 2014 se encontraban registradas 135 cooperativas de trabajo en la ciudad de Santa Fe, lo que representa el $88 \%$ del total de cooperativas. A su vez, verificando que las cooperativas de trabajo se constituyen generalmente durante coyunturas económicas recesivas, prácticamente la totalidad ha sido conformada luego de la crisis económico-política del año 2001.

Dentro de estas cooperativas podemos distinguir diferentes matrices de surgimiento: empresas recuperadas por sus trabajadores, cooperativas que nacieron de un impulso autónomo de los propios asociados, cooperativas promovidas a través de las ONG o de la acción de universidades, y por último, cooperativas inducidas por programas y políticas del Estado.

Desde el año 2010 en la Universidad Nacional del Litoral (UNL) se ha venido construyendo un espacio interdisciplinario orientado a la investigación, docencia, debate e intervención en el campo de la Economía Social y Solidaria (ESS) que fue permitiendo el surgimiento de diferentes proyectos de investigación y extensión, como así también de una catedra electiva para los estudiantes de grado de la Universidad. A partir de este espacio, frente al interés constante del equipo sobre el surgimiento y la dinámica de este tipo de emprendimientos asociativos, como también sobre el importante rol social que los mismos ejercen, surge en 2013 el Proyecto de Extension de Interés Social (PEIS) ${ }^{1}$ "Fortalecimiento y capacitación de las cooperativas de trabajo y otras organizaciones sociales en el marco de la economía social y solidaria en la ciudad de Santa Fe".2

\section{Cooperativas participantes y objetivos del proyecto de extension}

De acuerdo con Vuotto (2011), al valorizar el papel del trabajo como espacio de inserción y reconocimiento social y considerar el lugar central que ocupa en la existencia de las personas y la estructuración de la vida social, este tipo de iniciativas surge frente a la necesidad, por parte de sus miembros, de asegurarse un empleo que reúna entre otros atributos la estabilidad, cierto grado de seguridad y la posibilidad de alcanzar un ingreso. Desde esa óptica, resulta importante la supervivencia de la organización que se crea, así como el logro de una rentabilidad económica como garantía necesaria para la continuidad del empleo. En su momento, entendimos que desde la práctica extensionista podíamos aportar nuestro compromiso y trabajo para la sostenibilidad de estas organizaciones con las que veníamos articulando acciones aisladas desde 2011.

Así fue que el trabajo del equipo extensionista se focalizó en las siguientes cooperativas de trabajo: "Jóvenes por San Cayetano" y "Por un mañana mejor". Son cooperativas legalmente constituidas por separado pero que operativamente trabajan en forma conjunta

1) Aprobado por Res. CS de la UNL 410/13. Período de ejecución: junio 2013/junio 2015.

2) El presente PEIS tiene como antecedente el Proyecto de Investigación
CAI+D 2010 "Las cooperativas de trabajo en la ciudad de Santa Fe y su posibilidad de desarrollo en el marco de la Economía Social y Solidaria", cuyo director fue el Lic. Julio Tealdo. 


\author{
el Proyecto de Extensión de Interés \\ Social se propuso asistir al proceso \\ de autonomía, aprendizaje y \\ autogestión, organizando espacios \\ colectivos de capacitación, \\ asesoramiento y cooperación
}

y asociativa. El surgimiento formal de las cooperativas data del año 2005. Las mismas emergen del Plan Federal de Emergencia Habitacional de la Nación. La Cooperativa Eco-Hogar tuvo su origen cuando algunos de los actuales miembros eran beneficiarios de planes sociales $y$, motivados por encontrar alternativas a la falta de trabajo en el año 2005, se acercaron al Centro de Investigación y Desarrollo para la Construcción y Vivienda (CECOVI) de la Universidad Tecnológica Nacional - Facultad Regional Santa Fe guiados por un espíritu cooperativista e intentando sostener una idea que venían desarrollando en trabajos anteriores. En 2011 se conformaron formalmente como cooperativa. La Cooperativa Parque Bahía, en sus orígenes fue una empresa familiar que llegó a tener hasta 60 empleados en relación de dependencia y que funcionó por más de 20 años. En la crisis de 2001 la empresa familiar debió cerrar sus puertas. Diez años después, sus ex propietarios y familiares de éstos conformaron formalmente la cooperativa. Las cooperativas de trabajo mencionadas pertenecen a la rama de la construcción y han surgido motivadas por políticas públicas o bien por iniciativa propia en base a las convicciones de sus fundadores. El contacto con el equipo del PEIS provino de haber participado ambas partes en congresos y foros de ESS y en trabajos de campo solicitados desde la cátedra electiva de ESS de la UNL.

La mayor parte de lo que producen las cooperativas es demandada por el Estado y sólo una pequeña proporción por el sector privado, generando una dependencia importante hacia los organismos públicos. Los productos y servicios que generalmente producen y comercializan son: reparación y mantenimiento de estructuras edilicias, pavimento articulado y su colocación, bloques de cemento, entre otros.

\section{Objetivos principales del Proyecto de Extensión de Interés Social}

En primer lugar, se propuso asistir al proceso de autonomía, aprendizaje y autogestión, organizando espacios colectivos de capacitación, asesoramiento y cooperación. En segundo lugar, se planteó contribuir al fortalecimiento institucional y a la capacidad de gestión de las cooperativas de trabajo con los organismos públicos claves que tienen incidencia en su desarrollo económico. Un objetivo interno del equipo fue formar y consolidar una masa crítica de estudiantes y profesionales extensionistas en el ámbito de la UNL, desde un abordaje interdisciplinario, que permita desarrollar capacitaciones, asesorías y asistencias técnicas de diferentes temáticas tales como: ESS, análisis de costos, comercialización, cooperativismo, aspectos jurídicos sobre cooperativismo, entre otros.

\section{Identificación de problemáticas a resolver}

El trabajo de identificar las problemáticas de las cooperativas inherentes a sus aspectos contables y administrativos se facilitó debido a que la mayoría de los miembros del equipo extensionista proviene del campo de las ciencias económicas.

Se detectó que las cooperativas tenían serias dificultades en la gestión y organización de sus actividades productivas, administrativas y comerciales. Las condiciones socioeconómicas en las que desarrollaban sus actividades (competencia directa en el mercado) llevaban a que dichas organizaciones deban disponer o construir una organización mas ordenada y con mayor formación o capacidad profesional para que puedan tomar decisiones e 
implementar acciones en forma veloz y flexible; fortaleza y habilidad financiera para poder responder a situaciones cambiantes y escala física y logística que le permitan crecer en ámbitos locales y regionales. Es decir, tenían que funcionar a la par de una empresa capitalista que persigue la maximización de su utilidad.

En esta instancia, las cooperativas de trabajo presentaban varios inconvenientes. En gran parte de éstas los asociados ejercían tareas administrativas, comerciales y operativas, aunque la mayoría de ellos se encontraban realmente capacitados para la intervención en los procesos productivos.

Para todas las cooperativas en cuestión era sumamente necesario iniciar un proceso de fortalecimiento intraorganizacional y comercial. Las mismas demandaban capacitaciones en lo que respecta a temáticas específicas, como comercialización, administración, costos y planificación; colaboración y asistencia técnica para el diseño e implementación de instrumentos organizacionales que permitieran dinamizar el funcionamiento y organización interna de la cooperativa; aprehensión sobre modelos de gestión ligados a mecanismos asociativos que favorezcan procesos colectivos (cooperativismo, gestión asociada); aprendizajes en torno a instrumentos de participación ciudadana e incidencia en las políticas sociales (audiencias públicas, presupuesto participativo, trámites administrativos); obtención de saberes teóricos e instrumentales que les permitieran acceder a mecanismos de financiamiento y cooperación.

Por lo anteriormente enunciado, uno de los principales problemas que presentaban se manifestaba en la falta de capacidad para organizar y gestionar adecuadamente la cooperativa, además de las dificultades que presentaban a la hora de comercializar sus productos o servicios y de obtener nuevos clientes.

Las consecuencias que estas vicisitudes traían aparejadas se revelan en las siguientes dimensiones:

- Administración: carencia de conocimientos necesarios para poder llevar al día los trámites administrativos que los distintos entes gubernamentales les exigían. Asimismo tenían graves problemas para definir sus objetivos, actividades, responsabilidades y recursos.

- Costos: los asociados de las cooperativas no sabían exactamente el valor de los productos o servicios que ofrecían. Generalmente lo obtenían en forma general, lo que generaba una infravaloración de su mano de obra y de sus productos. A la hora de presentarse a licitaciones públicas o para presentar presupuestos en la parte privada presentaban precios menores a lo que realmente valía el trabajo a realizar.
- Comercialización: se focalizaban en un determinado tipo de cliente y de producto, lo que generaba una gran dependencia con los mismos. Además, a la hora de pensar la comunicación de la institución y de sus productos solían centrarse en las recomendaciones de clientes anteriores o allegados, dejando de lado las nuevas formas de comunicación virtuales (página Web, redes sociales) u otras más tradicionales (folleto institucional, radios comunitarias, entre otros).

- Asociativismo: las cooperativas no tenían una adecuada organización interna ya que los asociados no conocían la estructura de propiedad de la cooperativa, los derechos y obligaciones de cada uno ellos, ni los órganos que la componen y su adecuado funcionamiento. Tampoco disponían de reglamentos internos concertados democráticamente y aceptados por los miembros trabajadores.

\section{Metodología de la intervención}

Durante las primeras semanas de implementación del proyecto se realizaron distintos encuentros de trabajo entre los miembros del PEIS y los referentes de las cooperativas de trabajo involucradas. Estas reuniones se llevaron a cabo por separado y en las instalaciones de las cooperativas. Las primeras sirvieron para explicar claramente cuáles eran los objetivos, la finalidad y las actividades que se planeaban cumplir en los próximos meses, sin perder de vista que los protagonistas del proyecto eran los asociados. Esto último nos llevó, indudablemente, a considerar sus opiniones para la reformulación de objetivos y actividades. De esta forma se pudo crear un marco de confianza lo suficientemente sólido como para que todos los actores involucrados se comprometieran a participar en las actividades propuestas desde el equipo extensionista.

En las reuniones siguientes se efectuó un análisis colectivo de los inconvenientes que habían venido afrontando, de las problemáticas que tenían por resolver, analizamos su situación legal, impositiva y la de los asociados, entre otras temáticas que fueron surgiendo a lo largo de dichos encuentros.

De esta manera se logró recopilar la información necesaria para hacer un diagnóstico situacional y organizacional de cada una de las cooperativas.

Luego, se realizó una primera sistematización de la información relevada. Los temas predominantes que se detectaron para su análisis específico y posterior fortalecimiento fueron: la organización interna del trabajo, distribución de roles y responsabilidades, situación económica y financiera, comercialización de los productos 
y servicios ofrecidos, la posibilidad de recibir transferencias/ subsidios del Estado y la relación con sus representantes, estado del equipamiento y la necesidad de inversiones.

Vale destacar que se tuvieron en cuenta los comentarios de los asociados sobre aquellas cuestiones que, a su entender, debían ser atendidas con urgencia. Desde este enfoque, este proyecto se enmarcó en el campo de la Investigación-Acción Participativa (IAP). ${ }^{3}$ A continuación, se organizaron talleres de debate e intercambio de información con los actores públicos involucrados y las cooperativas de trabajo. Entre los actores públicos asistieron representantes de la Municipalidad de la ciudad de Santa Fe, del Ministerio de Trabajo y del Ministerio de Desarrollo Social del gobierno de la provincia de Santa Fe.

Se realizaron estos encuentros para reunir a las partes que tenían cuestiones e inconvenientes para ser resueltos (cooperativas de trabajo) con aquellas que tienen la responsabilidad o que podían estar en condiciones de aportar alguna solución a dichos problemas (Estados provincial, municipal y Universidad).

Uno de los objetivos sobresalientes de estos encuentros fue el de crear el ámbito acorde para que los integrantes de las cooperativas se conozcan y reconozcan como actores de la ESS local para así poder tratar de forma articulada y colectiva el fortalecimiento de este sector en la ciudad.

Posteriormente comenzaron los talleres de trabajo específicos con los asociados para miras de abordar los temas problemáticos mencionados anteriormente.

Dichos encuentros se basaron en la identificación de sus debilidades y fortalezas para afrontar dichas dificultades, la transferencia de herramientas técnicas administrativas por parte del equipo extensionista, la puesta en práctica de las mismas y, por último, el desarrollo conjunto de una estrategia que busque darle una solución estructural a los problemas operativos, administrativos y de gestión identificados.

\section{Vinculación con las instituciones participantes del PEIS}

Con el objetivo de sumar y coordinar esfuerzos y recursos disponibles, esta propuesta de trabajo interinstitucional tuvo con la ayuda y colaboración de organismos públicos relacionados con la temática que se propuso abordar desde el PEIS.

Las instituciones que vienen acompañando a la UNL en este proyecto son:

- Subsecretaría de Economía Social del Gobierno de Santa Fe: trabajó en forma articulada y conjunta con el equipo del PEIS de la
UNL en el Centro de Emprendedores de la Economía Social de la ciudad de Santa $\mathrm{Fe},{ }^{4}$ coordinando y participando en muchas de las actividades relativas a las capacitaciones y encuentros realizados junto a las cooperativas.

- Ministerio de Trabajo del Gobierno de Santa Fe: este organismo aportó los fondos necesarios para que una de las cooperativas pudiera diseñar, instalar y poner en marcha una fábrica de bloques de cemento y participó en el diseño del Plan de Trabajo base de la misma junto a miembros del equipo del PEIS.

- Dirección Ejecutiva de Economía Social de la Municipalidad de la ciudad de Santa Fe: esta área ha trabajado en forma mancomunada con la UNL en los últimos años en lo referente a capacitaciones, asesoramientos y asistencias técnicas requeridas tanto por emprendimientos socioproductivos como por emprendedores sociales, proporcionando recursos financieros y físicos (espacios y lugares para los encuentros de formación).

\section{Cambios efectivamente producidos}

Entre los principales cambios y resultados se pueden mencionar los siguientes: consolidación de la capacidad de autogestión de los asociados participantes (costos, comercialización, registración y distribución de tareas, obligaciones y derechos); afianzamiento institucional de las cooperativas de trabajo en su articulación con la UNL y los organismos públicos involucrados en el proyecto, y generación de un espacio colectivo de debate e intercambio de ideas sobre el cooperativismo de trabajo en la ciudad de Santa Fe. Esto último les permitió tomar conciencia de que las problemáticas que afectan a las cooperativas de trabajo son muy similares, llevándolos a reconocerse como pares y reforzando así el vínculo entre las cooperativas de la ciudad.

Por otro lado, se han gestionado y efectivizado subsidios por parte de los organismos públicos involucrados para instalar una fábrica de ladrillos de cemento, para la realización de capacitaciones sobre legislación cooperativa y para adquirir equipos y herramientas de trabajo.

También se consideran como cambios efectivos el empoderamiento e identificación de los asociados como integrantes del movimiento de la ESS. Este proceso de trabajo colectivo ha posibilitado que los miembros de las cooperativas de trabajos se vean a sí mismos como los verdaderos protagonistas del sector. A la par de este reconocimiento, han tomado conciencia de que para sostenerse en el tiempo como empresas cooperativas es necesario el fortalecimiento y consolidación institucional y organizacional de sus emprendimientos.
3) IAP: Método de investigación y aprendizaje colectivo de la realidad basado en un análisis crítico con la participación activa de los grupos implicados, que se orienta a estimular la práctica transformadora y el cambio social. Fuente: http://www.dicc.hegoa. ehu.es/listar/mostrar/132
4) Surge de un convenio firmado en julio de 2012 por la UNL, la Municipalidad de Santa Fe y el Gobierno de la provincia de Santa Fe. 

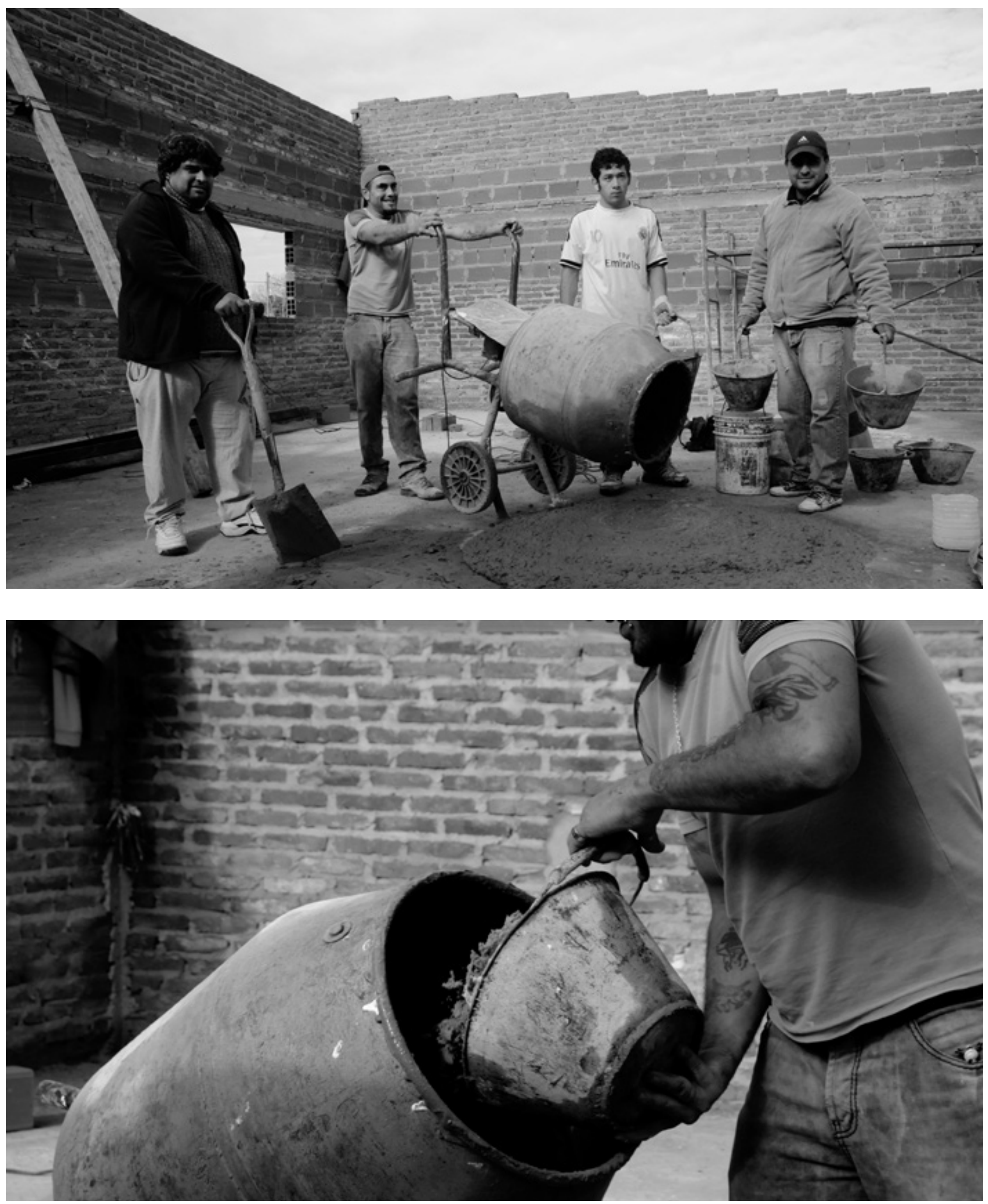
Además, han reconocido la importancia fundamental que tiene generar y tener trabajo decente, es decir, empleos de calidad, con cobertura de la protección social y respeto por los derechos fundamentales en el trabajo. Éste es uno de los desafíos más importantes a superar que se han planteado los asociados luego del trabajo de estos años.

\section{Conclusiones}

El equipo extensionista ha tenido la posibilidad de diseñar herramientas de trabajo innovadoras y creativas, puesto que el proceso de adaptación de los saberes técnicos fue un desafío importante a superar. Asimismo, estos dos años de trabajo articulado permitieron estrechar las relaciones humanas entre con los asociados y generar un vínculo de confianza para seguir avanzando y enfrentando nuevos desafíos.

Es importante destacar la necesidad de incorporar al equipo profesionales y estudiantes de disciplinas ligadas a aspectos productivos y sociales para ampliar la visión y el trabajo interdisciplinario que haga factible abordar en forma integral las problemáticas e iniciativas que surgen de las cooperativas y evitar la focalización exclusiva en los aspectos contables y administrativos. El diseño, ejecución y evaluación de un proceso interinstitucional, con el compromiso y esfuerzo de funcionarios públicos, referentes y asociados de las cooperativas de trabajo, coordinados por el equipo extensionista del PEIS, contribuyó a poder llevar a cabo los encuentros y talleres de trabajo con los integrantes de los entes asociativos según lo planificado.

A través de los encuentros desarrollados, los asociados no sólo obtuvieron capacidades y herramientas de gestión nuevas, también despertaron en su interior la voluntad necesaria para seguir adquiriendo otras habilidades y para replicar estos conocimientos en su comunidad, de manera de compartir sus experiencias con otras personas.

Destacamos que los asociados de las distintas cooperativas han tenido un proceso de empoderamiento en el campo de la ESS que nunca habían experimentado. En palabras de ellos: "Ahora nosotros nos sentimos parte y nos vemos dentro de la Economía Social y vamos a seguir construyendo y defendiendo esto que hemos venido levantando entre todos".

Seguramente también hubo momentos de crisis y de menor compromiso entre los involucrados en el proyecto. En los comienzos, la coordinación de las actividades a realizar tuvo sus dificultades, puesto que los referentes de las cooperativas no asistían a los encuentros de trabajo, o al equipo del PEIS se le hacía imposible contactarlos por varios días. Asimismo, en algunos casos los asociados mostraban desconfianza ante el trabajo propuesto desde el equipo, en tanto expresaban "no lo veo como algo útil para mi vida" y "no entendemos por qué la Universidad quiere trabajar con nosotros". Luego de los primeros meses de trabajo, podemos afirmar que esa desconfianza se vio superada y el compromiso de los asociados fue creciendo.

Entendemos que para avanzar en el trabajo extensionista con este tipo de organizaciones asociativas es necesario dejar de lado los caminos habituales, los saberes y las prácticas ya conocidas y sabidas, y comenzar a experimentar formando parte directamente de este camino que día a día se va abriendo paso en la cotidianidad de cada uno de nosotros. En el desarrollo del PEIS, por muchos momentos, hemos estado a la altura de semejante desafío, lo cual nos llena de gran satisfacción. Pero a su vez nos interpela como miembros de la universidad pública para que nuestro compromiso sea aún mayor y podamos redoblar esfuerzos para abarcar a más cantidad de experiencias cooperativas que se encuentran en la misma situación de estas cooperativas hace unos pocos años. Para finalizar, la tarea incansable de los asociados de las cooperativas de trabajo con las cuales se ha venido trabajando en el marco del PEIS demuestra que otra forma de hacer economía (o de participar en la economía) es posible y que ello no implica necesariamente perseguir en forma constante el lucro, éste debe convertirse en el medio para alcanzar un fin superior: que cada ciudadano lleve a cabo su vida de una forma digna, en correspondencia con las demás personas y con el medio en el cual convivimos.

\section{Referencias bibliográficas}

Cracogna, D. (2002). Crisis empresarias y cooperativas de trabajo. T. XIV. Buenos Aires: Errepar

Vuotto, M. (2011). El Cooperativismo de Trabajo en la Argentina: contribuciones para el diálogo social. Lima: OIT/Programa Regional para la Promoción del Diálogo y la Cohesión Social en América Latina (Serie Documento de Trabajo, 217). 